on the plasticity of neurodevelopmental disorder.

Klin and others suggest that there are cognitive deficits in assigning salience, which leave a person ill-equipped to set priorities and learn from experience. This has a significant bearing on any educational or therapeutic interventions.

The overarching cognitive theories are discussed in terms of experimental paradigms in an attempt to establish whether there may be a 'cognitive style' unique to autism. The fact of 'weak coherence' (Frith), seemingly at odds with the 'empathizingsystemizing' theory (Baron-Cohen), emphasises the need for more studies on the neurobiological hard-wiring underpinning deficits.

The importance of the five interconnected systems constituting the 'social brain', and the identification of fusiform face area as a possible 'neurofunctional marker' are exciting. They need to be balanced against the study of the amygdala which cautions that structural abnormalities may not always be reflected in physiological dysfunction. The use of nonverbal paradigms such as movement disorders and crossovers from non-autistic populations illustrate the many ways of skinning the neurobiological cat.

For me, this book has been as much an exploration of the mind of the researchers as of people with autism. It will probably age with grace in an electronic world and be of interest to clinicians and specialists in autism, given the range of disciplines represented, the international tenor and the evolving nature of the issues themselves. The glossary of acronyms at the end of each chapter was the only minor distraction in an interesting and instructive read.

Anupama lyer Specialist Registrar in Psychiatry of Learning Disabilities and Child Psychiatry, The Elms Health Centre, Slade Road, Halesowen, West Midlands B63 2UR, UK

\section{Cognitive-Behavior Therapy}

Edited by Jesse H.Wright. Washington, DC: American Psychiatric Association. 2004. 168 pp. $€ 23.50$ (pb). ISBN I 58562 I78 I

This brief edited book forms part of the 'Review of Psychiatry' series edited by John M. Oldham and Michelle B. Riba. There are five chapters in total: cognitive-behavioural therapy (CBT) for schizophrenia, CBT for bipolar disorder, computer-assisted CBT, CBT for patients with physical illness and CBT for children and adolescents. Each chapter is written clearly, with a range of clinical examples, tables and diagrams that bring the subject matter to life. The everyday practice of CBT is clearly in the mind of the authors, as is the importance of evaluating the evidence base. Each of the areas covered has shown an enormous rise in both research and practice over the past decade, and they hold great potential for the future. Therefore, reviews of this kind are extremely timely and relevant. Be warned, however, that the book does not stand alone as a reference because of its limited breadth. For example, at the front there is an overview of the chapters within the book but there is no introductory chapter on CBT to set the groundwork of its principles and practice across a wide range of presenting problems. The limited coverage also makes the title of the book slightly misleading and its cost slightly too high. Nevertheless, I would recommend that health practitioners involved in these emerging areas read the well-researched, accessible chapters in this book now while they still represent the cutting edge of contemporary CBT.

Warren Mansell Clinical Research Psychologist, Department of Psychological Medicine, Institute of Psychiatry, De Crespigny Park, London SE5 8AF, UK

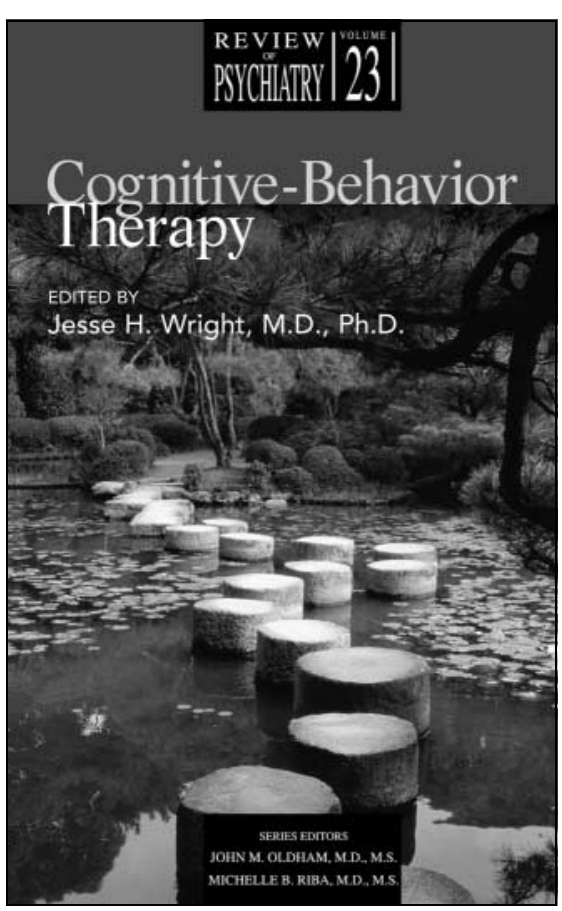

Talking Over the Years: A Handbook of Dynamic Psychotherapy with Older Adults

Edited by Sandra Evans \& Jane Garner. Hove and New York: Brunner Routledge. 2004. 280 pp. $€ 17.99$ (pb).

ISBN I 5839| 1448

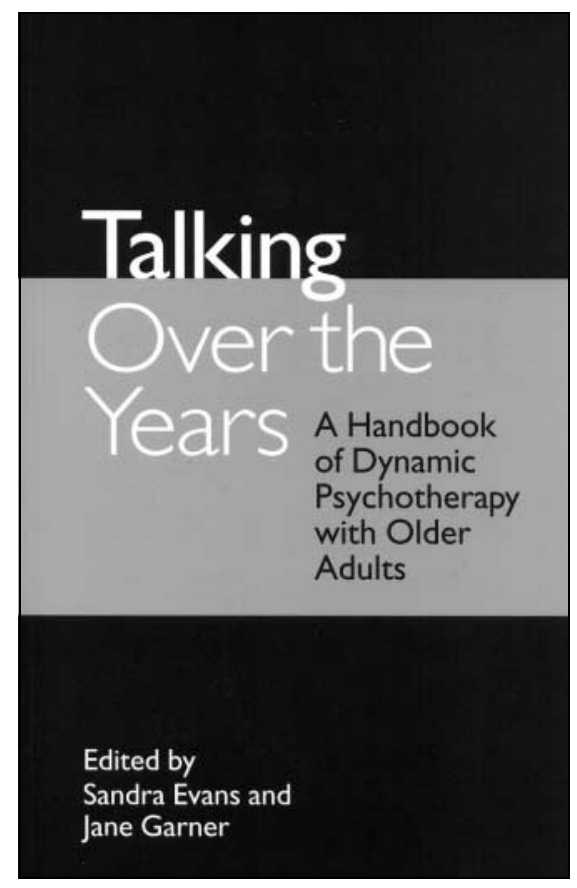

This book presents a wide range of material on the mental health of older people from a psychodynamic perspective. It provides information about the lives and theories of major psychoanalytic and psychodynamic thinkers and considers the application of their ideas to the understanding of later life. Psychodynamics is discussed in relation to work with patients as well as in relation to staff. Many apposite case illustrations are included. The book contains chapters about a range of methods of working psychotherapeutically with people with and without dementia, including not only one-to-one, family and group talking therapies but also expressive approaches using other media for communication.

The amazing breadth of this book may be seen as both its strength and its weakness. On the one hand it provides a stimulating cocktail, but on the other this detracts from a clear focus. Those who might buy the book for its subtitle could be disappointed to find that this is not a guide on 'how to do' psychotherapy: although the excellent chapter on brief therapy does provide some nitty-gritty advice and those 